\title{
Comprehensive Molecular Analysis of Serologically D-Negative and Weak/Partial D Phenotype in Thai Blood Donors
}

\author{
Jairak Thongbut ${ }^{a, b} \quad$ Loann Raud $^{c, d}$ Claude Férec $^{c-e} \quad$ Charuporn Promwong $^{\text {b }}$ \\ Pornlada Nuchnoj ${ }^{a, f}$ Yann Fichou ${ }^{c, d}$ \\ aDepartment of Clinical Microscopy, Faculty of Medical Technology, Mahidol University, Bangkok, Thailand; \\ ${ }^{b}$ National Blood Centre, Thai Red Cross Society, Bangkok, Thailand; ' Univ Brest, INSERM, EFS, UMR 1078, GGB, \\ Brest, France; ' ${ }^{L}$ aboratoire de Génétique Moléculaire et d'Histocompatibilité, CHU Morvan, Brest, France; \\ e Laboratory of Excellence GR-Ex, Brest, France; ${ }^{\mathrm{f} C e n t e r}$ for Research and Innovation, Faculty of Medical Technology, \\ Mahidol University, Bangkok, Thailand
}

\section{Keywords}

Blood group $\cdot$ Genotype $\cdot$ Phenotype $\cdot \mathrm{Rh} \cdot \mathrm{RHD}$ variants

\begin{abstract}
Background: Molecular genetics of the Rh system has been extensively studied in Caucasians, Black Africans, East Asians, and Indians more recently. In this work, we sought to investigate the molecular basis of variant $D$ expression in the Thai population, which remains unknown. Materials and Methods: Blood samples from 450 Thai donors showing the variant D phenotype were collected. The RHD gene was analyzed by quantitative multiplex polymerase chain reaction of short fluorescent fragments and/or Sanger sequencing. Results: The most frequent alleles in $200 \mathrm{D}$-negative and $121 \mathrm{DEL}$ samples were the whole RHD gene deletion and the Asian DEL alleles, respectively. In 129 weak/partial D samples, 36 variant alleles were identified, including eight novel alleles. $R H D^{*} 06.03$, which is common in variant $\mathrm{D}$ samples from South China, is the most prevalent variant allele, followed by the recently reported Indian RHD*01W.150 allele. Discussion: For the first time, a comprehensive overview of the nature and distribution of variant $R H D$ alleles in Thailand is reported. It is a milestone to pave the way towards improvement of the current screening strategy to identify DEL donors accurately. The next step will be the design and implementation of a simple molecular test for screening the most frequent alleles, specifically in this population.

( 2019 S. Karger AG, Basel
\end{abstract}

\section{Introduction}

Antigen expression in the $\mathrm{Rh}$ system, which is the most complex and polymorphic blood group system, is driven by two homologous $R H$ genes: $R H D$ and $R H C E$ [1]. The most clinically relevant antigen in this system, antigen $\mathrm{D}$, is encoded by the former gene. Anti-D alloimmunization can occur in D-negative patients when exposed to D-positive red blood cells and may be responsible for hemolytic transfusion reaction and hemolytic disease of the fetus and newborn with severe clinical outcomes [2].

Variations in D phenotype expression in Caucasians, Black-Africans, and East-Asians, as well as the molecular genetics of $R H$ genes, have been extensively studied and described, and the global distribution of variant $\mathrm{RH}$ alleles is well known in these populations [3-6]. In Western and East-Asian countries, molecular typing; i.e., genotyping, in addition to serological study, has become routine practice to assess the risk of alloimmunization [7, 8] and is therefore a critical step in the whole management of transfusion and pregnancy at risk.

A key point in molecular RHD typing is allele distribution, which is known to vary largely between ethnicities. The knowledge about the molecular background of

Pornlada Nuchnoi and Yann Fichou contributed equally to this work. 
the $R H D$ gene on a regional/national basis is necessary to implement an efficient, dedicated genotyping strategy. For example, Caucasian-specific tests for routine diagnostics have been developed and implemented to identify preferentially the $R H D^{*}$ weak $D$ type 1 , $R H D^{*}$ weak $D$ type 2 , and $R H D^{*}$ weak $D$ type 3 alleles [912], which have long been known to be the most common variant alleles in serologic weak $D$ individuals of Caucasian origin [3]. This latter strategy would be definitely inefficient for typing people of African or Asian origin. In Korea, Seo et al. [13] designed and implemented an effective diagnostic strategy for the detection of $R H D$ variants in serologically $\mathrm{D}$ - Korean donors, including the typical Asian DEL allele characterized by the splice site variation c.1227G $>$ A [14], which is the most prevalent $D E L$ allele in the East-Asian population $[6,15$, 16]. Extensive molecular data are available in Caucasians, Black Africans, East-Asians, and Indians since more recently. Conversely, populations of other origins, such as Thais, have been barely documented. It is worth mentioning that preliminary studies have been conducted to investigate the $\mathrm{Rh}$ blood group system in the Thai population. As early as in 2002 , a large-scale study reported that $99.69,0.30$, and $0.01 \%$ of the Thai blood donors are $\mathrm{D}_{+}, \mathrm{D}_{-}$, and weak $\mathrm{D}$, respectively [17]. Later, another investigation in samples with serological weak D expression showed that partial DVI, which is clinically relevant, is the most common phenotype $(62 / 81$, $76.5 \%)$ in this subset of samples [18]. In terms of molecular analysis, the Asian DEL allele was found in 18.9\% of the serologically D- samples [19], and a recent study also reported preliminary $R H$ genotyping data in a subset of nonselected donors from North and Central Thailand [20]. Overall, few studies have been carried out in Thailand, and the molecular determinants of Rh D variability, which is currently unknown in this population, remain to be described in detail at the genetic level. Here, we report for the first time a comprehensive study investigating the molecular basis of those variant $\mathrm{D}$ samples in the Thai population.

\section{Materials and Methods}

\section{Blood Sample Collection and Serological Analysis}

Blood samples from unrelated donors from Bangkok and suburban provinces were collected and provided by the National Blood Centre (NBC), Thai Red Cross Society (Bangkok, Thailand). Blood samples were analyzed routinely with automate analyzer PK7300 Microplate Agglutination Systems (Beckman Coulter) with monoclonal anti-D (clones LDM2 and RUM-1, NBC). Samples with inconclusive or negative results in $\mathrm{D}$ typing were retyped with two commercial blended IgM/IgG monoclonal antiD reagents: IgM clone LDM2 and IgG clone LHM77 (NBC), IgM clone RUM-1 and IgG clone MS-26 (Plasmatec, Bridport, UK). Using conventional tube test at room temperature, $37^{\circ} \mathrm{C}$, and in- direct antiglobulin test (IAT), 450 donor samples were classified either as weak/partial D or D negative. Weak/partial D samples showed either agglutination reaction $\leq 2+$ or IAT positive. Rh CcEe typing was carried out in all donor samples by using inhouse anti-c and -E (NBC), and commercial anti-C and -e (DiaClon; DiaMed GmbH, Bio-Rad Laboratories) monoclonal IgM antibodies.

IAT-negative samples were subsequently screened for DEL by the adsorption-elution technique with monoclonal anti-D reagent (NBC). Briefly, $200 \mu \mathrm{L}$ of donor red blood cells were washed with normal saline solution $(0.9 \%)$ and mixed with equal volume of anti-D reagent. The mixture was incubated at $37^{\circ} \mathrm{C}$ for $1 \mathrm{~h}$ and then washed thoroughly with normal saline solution. The eluate was prepared by the in-house $0.1 \mathrm{M}$ glycine- $\mathrm{HCl}$ buffer/ 10\% EDTA (V/V: 4:1) technique as previously described [21]. Eluate and last-washed supernatant were used for IAT (conventional tube test) against $\mathrm{D}$-positive and $\mathrm{D}$-negative control cells (NBC).

\section{DNA Extraction and Quantification}

Genomic DNA was extracted from whole blood by using commercial kits (BioFact Genomic DNA Prep Kit; BioFact, Celbridge, Ireland; PureDireX Genomic DNA Isolation Kit; Bio-Helix, Keelung City, Taiwan; or PureLink Genomic DNA Mini Kit; Thermo Fisher Scientific, Bangkok, Thailand). DNA concentration and purity $\left(\mathrm{A}_{260} / \mathrm{A}_{280}\right)$ were measured with a NanoDrop ${ }^{\mathrm{TM}} 2000 / 2000 \mathrm{c}$ Spectrophotometer (Thermo Fisher Scientific).

\section{RHD Genotyping}

Analysis by quantitative multiplex polymerase chain reaction (PCR) of short fluorescent fragments (QMPSF) was carried out to investigate potential exon copy number variations/rearrangements in the RHD gene by using the F9 and HFE genes for positive amplification control and copy number normalization in conditions previously described [22]. Basically, QMPSF is a simple multiplex, fluorescent PCR-based method, which aim is here to specifically identify $R H D$ exon markers to quantitatively assess exon copy number variations, which are frequently encountered at the $R H D$ locus, and to easily identify variant alleles, including hybrid $D-C E$ genes. Respective fragment length and peak areas were analyzed by GeneMapper v4.0 software (Applied Biosystems, Villebon-sur-Yvette, France). Exon 3 duplication allele was confirmed and screened in weak D samples by a previously reported PCRbased method [23]. Finally, when necessary, all ten RHD exons were amplified and directly sequenced, and sequencing data were analyzed by Sequencher v5.0 software (Gene Codes Corporation, Ann Arbor, MI, USA) [11]. Genotypes were deduced from both QMPSF and sequencing data on the basis of the most likely combination of variant $R H D$ alleles.

\section{Minigene Splicing Assay}

Minigene splicing assay was carried out to investigate the effect of an intronic variation on splicing as previously described [11, 23]. Briefly, both wild-type and mutated regions of RHD exon 8 and flanking intronic domains were PCR amplified. PCR products were inserted into the minigene to generate recombinant vectors that were subsequently transfected into HEK293 cells (ATCC Number CRL-1573). Cells were harvested $48 \mathrm{~h}$ after transfection. Total RNA was extracted and purified by the RNeasy Mini Kit (Qiagen, Courtaboeuf, France) and served as a template for reverse-transcription (RT)-PCR with the QIAGEN OneStep RTPCR Kit (Qiagen). Finally, RT-PCR products were visualized on a $2 \%$ agarose gel containing ethidium bromide and directly sequenced after gel extraction. 
Table 1. Serologic and $R H D$ genotyping data in 200 D-negative Thai blood donors

\begin{tabular}{lllllllrrr}
\hline Genotype $^{\mathrm{a}}$ & Mechanism & Exon & CCee & Ccee & CcEe & ccEe & ccee & Occurrence & \% \\
\hline RHD*01N.01 & Large deletion & $1-10$ & 6 & 39 & 1 & 4 & 100 & 150 & 75.0 \\
RHD*01N.03 & Hybrid gene & $3-9$ & 2 & 21 & - & - & - & 23 & 11.5 \\
RHD*01EL.01 & Splicing & 9 & 10 & 11 & - & - & - & 21 & 10.5 \\
RHD*01N.05 & Hybrid gene & $3-8$ & - & 2 & - & - & - & 2 & 1 \\
RHD*01N.61 & Nonsense & 7 & - & - & - & 1 & - & 1 \\
RHD*01N.83 & Frameshift & 5 & - & - & - & 1 & - & 1 \\
RHD-CE(3-10) & Hybrid gene & $3-9$ & - & 1 & - & - & - & 1 \\
RHD*05.04 & Missense & 5 & - & 1 & - & - & - & 1 \\
\hline Total & & & 18 & 75 & 1 & 6 & 100 & 200 \\
\hline
\end{tabular}

a Second allele is $R H D^{*} 01 N .01$. RHD*01N.01: whole RHD gene deletion; RHD*01N.03: RHD-CE(3-9)-D; RHD*01EL.01: RHD(c.1227G>A); RHD*01N.05: RHD-CE(3-8)-D; RHD*01N.61: RHD(c.952C>T); RHD*01N.61: RHD(c.702delG); RHD*05.04: $R H D($ c.697G $>$ C).

Table 2. Serologic and RHD genotyping data in 121 DEL Thai blood donors

\begin{tabular}{|c|c|c|c|c|c|c|c|c|}
\hline Genotype $^{a}$ & Exon & CCee & Ccee & CCEe & $\mathrm{CcEe}$ & ccee & Occurrence & $\%$ \\
\hline RHD*01EL.01 & 9 & 24 & 82 & - & - & 1 & 107 & 88.4 \\
\hline RHD*01N.01 & $1-10$ & - & 4 & - & - & 7 & 11 & 9.1 \\
\hline RHD*01N.03 & $3-9$ & - & 1 & - & - & - & 1 & 0.8 \\
\hline RHD*01N.16/RHD*01EL.01 & $3-8,9$ & - & - & 1 & - & - & 1 & 0.8 \\
\hline$R H D^{*} 15 / R H D^{*} 01 N .03$ & $3-9$ & - & - & - & 1 & - & 1 & 0.8 \\
\hline Total & & 24 & 87 & 1 & 1 & 8 & 121 & 100.0 \\
\hline
\end{tabular}

a Second allele is $R H D^{*} 01 N .01$, if not mentioned. $R H D^{*} 01 E L .01$ : $R H D(c .1227 \mathrm{G}>\mathrm{A})$; $R H D^{*} 01 N .01$ : whole $R H D$ gene deletion; RHD*01N.03: RHD-CE(3-9)-D; RHD*01N.16: RHD(c.711delC); $R H D^{*} 15: R H D($ c.845G >A).

\section{Results}

\section{Phenotypic Characterization of Thai Blood Donors with Variant D Phenotype}

In the 450 Thai blood donors, 129 and 321 samples were classified as weak/partial D and D negative by routine testing, respectively. Subsequent test by adsorptionelution in the 321 latter samples actually identified 121 DEL samples, while the other 200 samples were D negative. The RHD gene was then genotyped in all samples to identify the respective molecular bases of the variant $D$ phenotype.

Molecular Analysis of Serological D-Negative Samples Samples typed as D negative by serological analysis $(n=200)$ were first investigated by RHD QMPSF, which has proven useful for genotyping such samples. As commonly observed, whole deletion of the RHD gene at the homozygous state is the most frequent genotype (Table $1 ; 150 / 200,75.0 \%)$ notably found in all 100 ccee samples, followed by hybrid genes in 26 samples. RHD gene exons were subsequently sequenced in the remain- ing 24 samples. Interestingly, the Asian $D E L$ allele; i.e., $R H D(c .1227 \mathrm{G}>\mathrm{A})$, was found at the hemizygous state in 21 samples, while the other three samples carry singlenucleotide variations (Table 1).

\section{Molecular Analysis of Serological DEL Samples}

All 121 serological DEL samples were first screened in RHD exon 9. The Asian DEL allele was identified in 108 samples (Table 2, 89.2\%). QMPSF analysis and direct sequencing resolved twelve samples carrying negative $R H D$ alleles, and are thus supposed to be D negative, while a single sample was found to carry the RHD*weak-D type 15 allele (Table 2).

\section{Molecular Analysis of Weak-/Partial-D Samples}

The 129 weak/partial D samples were investigated by RHD QMPSF and/or direct sequencing. This analysis showed a large heterogeneity in terms of genotypes in the Thai population (Table 3). Indeed, as many as 52 allele combinations, including 33 observed only once, were found in those samples. The most common weak/ partial $D$ allele is partial $D V I$ type 3 (or $R H D^{*} 06.03$; al- 
Table 3. Serologic and RHD genotyping data in 129 weak/partial D Thai blood donors

\begin{tabular}{|c|c|c|c|c|c|c|c|c|}
\hline RHD allele 1 & RHD allele 2 & CCee & Ccee & $\mathrm{CcEe}$ & $\mathrm{ccEe}$ & ccee & Occurrence & $\%$ \\
\hline$R H D^{*} 06.03$ & RHD*01N.01 & 3 & 17 & - & - & - & 20 & 15.5 \\
\hline$R H D^{*} 06.03$ & RHD ${ }^{*} 01 E L .01$ & 6 & 1 & - & - & - & 7 & 5.4 \\
\hline RHD*06.03 & $R H D^{*} 01 N .03$ & 2 & - & - & - & - & 2 & 1.6 \\
\hline$R H D^{*} 18$ & $R H D^{*} 01 N .01$ & - & 4 & 1 & - & - & 5 & 3.9 \\
\hline RHD*18 & RHD*18 & 1 & - & - & - & - & 1 & 0.8 \\
\hline$R H D^{*} 18$ & RHD*01EL.01 & 1 & - & - & - & - & 1 & 0.8 \\
\hline RHD*01W.150 & $R H D^{*} 01 N .01$ & - & 6 & - & - & - & 6 & 4.7 \\
\hline$R H D^{*} 01 W .150$ & RHD*01EL.01 & 1 & 1 & - & - & - & 2 & 1.6 \\
\hline RHD*05.04 & $R H D^{*} 01 N .01$ & - & 5 & - & - & - & 5 & 3.9 \\
\hline RHD*0W.122 & $R H D^{*} 01 N .01$ & 1 & 3 & - & - & - & 4 & 3.1 \\
\hline RHD*0W.122 & RHD*01EL.01 & 1 & - & - & - & - & 1 & 0.8 \\
\hline$R H D(R 10 Q, V 174 M)^{\mathrm{a}}$ & $R H D^{*} 01 N .01$ & 1 & 3 & - & - & - & 4 & 3.1 \\
\hline$R H D(S 75 N)^{\mathrm{a}}$ & RHD*01N.01 & - & 3 & - & - & - & 3 & 2.3 \\
\hline$R H D(Q 89 P)^{\mathrm{a}}$ & $R H D^{*} 01 N .01$ & 1 & 2 & - & - & - & 3 & 2.3 \\
\hline RHD*08.01 & RHD*01N.01 & 3 & - & - & - & - & 3 & 2.3 \\
\hline RHD*15 & $R H D^{*} 01 N .01$ & - & - & - & 2 & - & 2 & 1.6 \\
\hline RHD*15 & RHD*01EL.01 & - & - & 1 & - & - & 1 & 0.8 \\
\hline RHD*01W.33 & $R H D^{*} 01 N .01$ & 1 & 1 & - & - & - & 2 & 1.6 \\
\hline RHD*01W.33 & RHD*01N.03 & 1 & - & - & - & - & 1 & 0.8 \\
\hline RHD*01W.33 & RHD*06.03 & 1 & - & - & - & - & 1 & 0.8 \\
\hline RHD*01W.25 & RHD*01N.01 & - & - & - & 2 & - & 2 & 1.6 \\
\hline RHD*01W.152 & RHD*01N.01 & - & 1 & - & - & - & 1 & 0.8 \\
\hline RHD*01W.152 & RHD*01EL.01 & - & 1 & - & - & - & 1 & 0.8 \\
\hline RHD*01W.152 & $R H D^{*} 06.03$ & 1 & - & - & - & - & 1 & 0.8 \\
\hline$R H D(G 307 E)^{\mathrm{a}}$ & RHD*01N.01 & - & - & - & - & 1 & 1 & 0.8 \\
\hline$R H D(G 307 E)^{\mathrm{a}}$ & RHD*01N.03 & - & 1 & - & - & - & 1 & 0.8 \\
\hline RHD*01W.6 & RHD*01N.01 & 1 & - & - & - & - & 1 & 0.8 \\
\hline RHD*01W.31 & RHD*01N.01 & - & - & - & 1 & - & 1 & 0.8 \\
\hline RHD*01W.72 & RHD*01N.01 & - & 1 & - & - & - & 1 & 0.8 \\
\hline RHD*01W.100 & RHD*01N.01 & - & - & - & 1 & - & 1 & 0.8 \\
\hline RHD*01W.119 & RHD*01N.01 & - & 1 & - & - & - & 1 & 0.8 \\
\hline RHD*01W.128 & RHD*01N.01 & - & - & - & - & 1 & 1 & 0.8 \\
\hline RHD*01W.154 & RHD*01N.01 & 1 & - & - & - & - & 1 & 0.8 \\
\hline$R H D(L 20 Q)^{\mathrm{a}}$ & RHD*01N.01 & 1 & - & - & - & - & 1 & 0.8 \\
\hline$R H D(L 110 P, F 223 V)^{\mathrm{a}}$ & RHD*01N.01 & 1 & - & - & - & - & 1 & 0.8 \\
\hline$R H D(S 230 N)$ & $R H D^{*} 01 N .01$ & - & - & - & 1 & - & 1 & 0.8 \\
\hline$R H D(K 394 T)^{\mathrm{a}}$ & RHD*01N.01 & 1 & - & - & - & - & 1 & 0.8 \\
\hline RHD*05.01 & RHD*01N.01 & - & 1 & - & - & - & 1 & 0.8 \\
\hline RHD*06.02 & RHD*01N.01 & - & - & 1 & - & - & 1 & 0.8 \\
\hline RHD*14.02 & RHD*01N.01 & - & 1 & - & - & - & 1 & 0.8 \\
\hline RHD*01W.10 & RHD*01EL.01 & 1 & - & - & - & - & 1 & 0.8 \\
\hline$R H D(P 221 L)^{\mathrm{a}}$ & RHD*01EL.01 & - & - & 1 & - & - & 1 & 0.8 \\
\hline$R H D(c .960 G>A)$ & $R H D^{*} 01 E L .01$ & 1 & - & - & - & - & 1 & 0.8 \\
\hline RHD*09.02.01 & RHD*01EL.01 & - & 1 & - & - & - & 1 & 0.8 \\
\hline RHD*17.04 & RHD ${ }^{*} 01 E L .01$ & 1 & - & - & - & - & 1 & 0.8 \\
\hline$R H D^{*} c .1153+6 T>C$ & RHD*01EL.01 & 1 & - & - & - & - & 1 & 0.8 \\
\hline RHD*01EL.01 & $R H D^{*} 01$ & 3 & - & - & - & - & 3 & 2.3 \\
\hline RHD*01EL.01 & RHD*01N.01 & - & 1 & - & - & - & 1 & 0.8 \\
\hline RHD*01EL.01 & RHD*01N.03 & 1 & - & - & - & - & 1 & 0.8 \\
\hline RHD*01 & RHD*01N.01 & 6 & 5 & - & - & 1 & 12 & 9.3 \\
\hline$R H D^{*} 01$ & $R H D^{*} 01$ & 6 & 1 & 1 & - & - & 8 & 6.2 \\
\hline RHD*01N.01 & RHD*01N.01 & - & - & - & - & 3 & 3 & 2.3 \\
\hline Total & & 50 & 61 & 5 & 7 & 6 & 129 & 100.0 \\
\hline
\end{tabular}

a Novel alleles. Refer to The Human RhesusBase v2.3 (URL: www.rhesusbase.info/) for details about the respective alleles. 
Table 4. Novel RHD alleles identified in the Thai population

\begin{tabular}{|c|c|c|c|c|}
\hline Designation & Nucleotide change (exon) & $\begin{array}{l}\text { Amino acid change } \\
\left(\text { position }^{\mathrm{a}}\right)\end{array}$ & $\begin{array}{l}\text { Most likely } \\
\text { haplotype }\end{array}$ & $\begin{array}{l}\text { GenBank } \\
\text { Accession No. }\end{array}$ \\
\hline RHD(R10Q,V174M) & c. $29 \mathrm{G}>\mathrm{A}(1), \mathrm{c} .520 \mathrm{G}>\mathrm{A}(4)$ & p.Arg10Gln (ICL1), p.Val174Met (M5) & $C D e$ & MK086934 \\
\hline RHD(L110P,F223V) & c. $329 \mathrm{~T}>\mathrm{C}(2)$, c. $667 \mathrm{~T}>\mathrm{G}(5)$ & p.Leu110Pro (M3), p.Phe223Val (M6) & $C D e$ & MK086935 \\
\hline RHD(L20Q) & c.59T $>$ A (1) & p.Leu20Gln (M0) & CDe & MK086936 \\
\hline$R H D(\mathrm{~S} 75 \mathrm{~N})$ & c. $224 \mathrm{G}>\mathrm{A}(2)$ & p.Ser75Asn (M2) & $C D e$ & MK086937 \\
\hline RHD(Q89P) & c. $266 \mathrm{~A}>\mathrm{C}(2)$ & p.Gln89Pro (M2) & $C D e$ & MK086938 \\
\hline$R H D(\mathrm{P} 221 \mathrm{~L})$ & c. $662 \mathrm{C}>\mathrm{T}(5)$ & p.Pro221Leu (M6) & $c D E$ & MK086939 \\
\hline RHD (G307E) & c. $920 \mathrm{G}>\mathrm{A}(6)$ & p.Gly307Glu (M9) & $c D e$ & MK086940 \\
\hline$R H D(\mathrm{~K} 394 \mathrm{~T})$ & c. $1181 \mathrm{~A}>\mathrm{C}(9)$ & p.Lys394Thr (ICL7) & $C D e$ & MK086941 \\
\hline
\end{tabular}

a Position in the RhD protein indicated in accordance with reference [24]. ICL: intracellular loop; M: transmembrane domain.

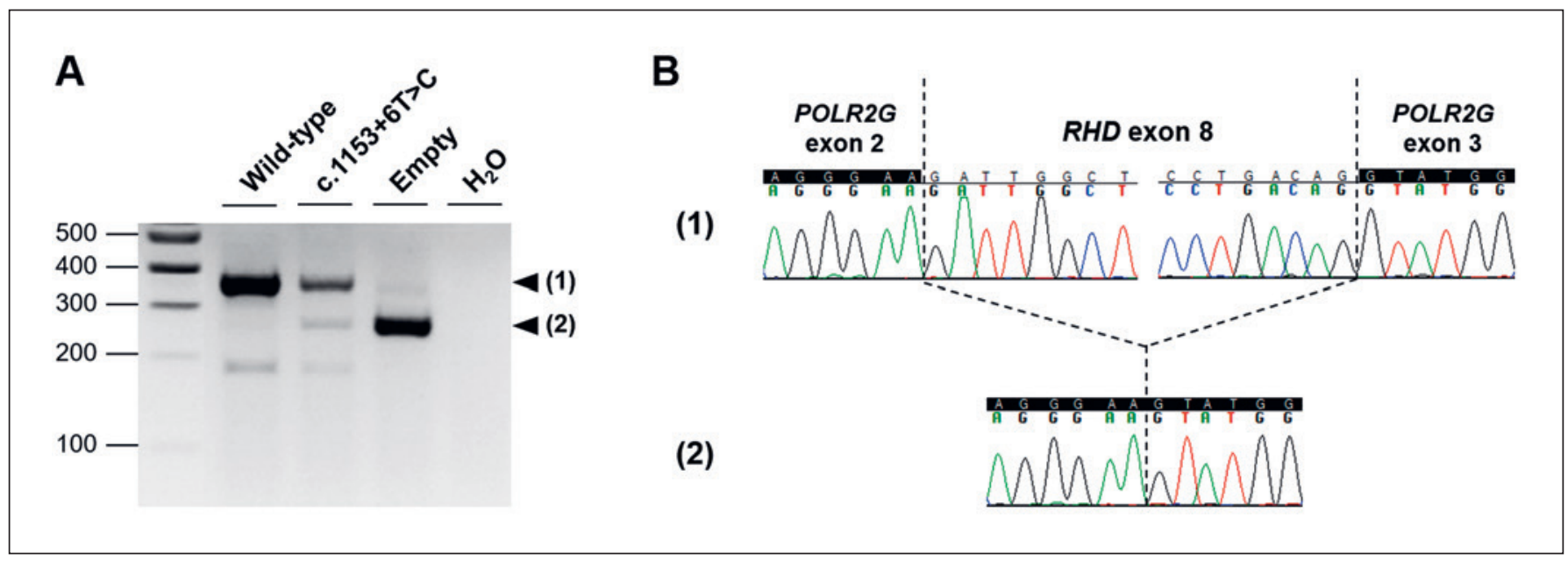

Fig. 1. Functional analysis of variant c. $1153+6 \mathrm{~T}>\mathrm{C}$ by minigene splicing assay. A RT-PCR profiles and $\mathbf{B}$ direct sequencing patterns showing (1) inclusion and (2) full skipping of RHD exon 8. 100 to 500: size in base pairs; Empty: empty vector (no insert); $\mathrm{H}_{2} \mathrm{O}$ : no template control.

lele frequency $=0.120)$. Interestingly, the most frequent weak $D$ allele $\left(R H D^{*}\right.$ weak $D$ type 150$)$ recently reported in the Indian population, which involves a duplication of a $\sim 12$ kilobase-region including RHD exon 3, was observed in 8 samples (6.2\%). Eight novel alleles (Table 4) were found in 16 donors, suggesting that the Thai population is heterogeneous in terms of RHD molecular genetics. Of those, both $R H D(\mathrm{R} 10 \mathrm{Q}, \mathrm{V} 174 \mathrm{M})$ and $R H D(\mathrm{~L} 110 \mathrm{P}, \mathrm{F} 223 \mathrm{~V})$ involve two variations already reported separately. The other six novel alleles involve single missense variations.

We next paid attention to a variant in intron 8, c. $1153+6 \mathrm{~T}>\mathrm{C}$ ( or IVS8+6T $>C$ ), which was previously reported (GenBank Accession No.: JX114750). To get insight into the functional defect induced by the intronic variant, we took advantage of our previously reported minigene assay, which has proven useful for the characterization of variants affecting splicing $[11,23]$. Our test clearly demonstrated that c.1153+6T $>$ C significantly al- ters normal splicing by promoting RHD exon 8 exclusion from the mature transcript (Fig. 1A). A "normal" fulllength transcript including exon 8 was though identified by sequencing analysis (Fig. 1B). Overall, this assay suggests that $\mathrm{c} .1153+6 \mathrm{~T}>\mathrm{C}$ is a splicing variant affecting $\mathrm{D}$ antigen expression quantitatively, but not qualitatively, and therefore is not of clinical importance.

\section{Discussion}

The nature and distribution of variant $R H D$ alleles are fundamental data for the Public Healthcare system to implement efficient and cost-effective genotyping tests and to guide population-specific transfusion practice and obstetrical management [24]. In order to get insights into the molecular determinants of variant $\mathrm{D}$ expression, we sought to investigate the RHD gene in the Thai population, which has remained globally unexplored so far. For 
the first time, we report a comprehensive overview of the variant $R H D$ alleles in this population.

As expected, whole $R H D$ gene deletion at the homozygous state is the most common $D$-negative genotype in serological D-negative Thai blood donors (Table 1, $75.0 \%)$. When considering only the subset of $\mathrm{C} / \mathrm{E}+$ samples $(n=100)$, frequency of this genotype is in the range of what has been reported before in other Asian populations (36.4-63.1\%) [15, 16, 25, 26]. Interestingly, as many as 21 samples out of 200 were found to carry the Asian $D E L$ allele. This result suggests that either another variation in $R H D$, which has not been identified yet, impairs $\mathrm{RhD}$ protein expression, or reagents and/or conditions for identifying DEL samples are not optimal, thus yielding false-negative results. In this more likely latter hypothesis, DEL screening methodology should be reviewed urgently in a large-scale study including genotyped samples. Unexpectedly, one sample found to be $\mathrm{D}$ negative serologically was genotyped as $R H D^{*} 05.04$. This finding may be due to either the absence of some $\mathrm{D}$ epitopes recognized by the antibodies used in this study as previously reported [27], leading to a "false" apparent D-negative phenotype, or another unidentified deleterious variation in the $R H D$ gene that impairs $\mathrm{D}$ antigen expression.

As also expected, $R H D(\mathrm{c} .1227 \mathrm{G}>\mathrm{A})$ is the most frequent $D E L$ allele found in $\sim 90 \%$ of the samples (Table 2 ). Based on the genotyping data, in this group of serologic DEL samples, the percentage of false-positive results is significant (Table 2; 12/121, 9.9\%), confirming that the methodology for DEL sample identification should be revised.

Finally, as many as 36 variant $R H D$ alleles were found in 129 serological weak/partial D samples, highlighting the large variability of the $R H D$ gene in the Thai population. Eight novel alleles were found, thus contributing to extend the molecular catalogue of $R H D$ polymorphism. It also suggests that many other novel alleles are very likely to be found in future studies in the Thai population. The most frequent allele is $R H D^{*} 06.03$, which was also reported to be the most frequent in $\mathrm{D}$ variant donors from South China $(28 / 62,45.2 \%)$ [28]. Very interestingly, the second most common variant allele is $R H D^{*} 01 W .150$, which was recently reported in the Indian population [29]. Taken together, besides the population-specific pattern observed in Thais, those data highlight the mixed nature of this population and, in parallel with the geographical context, provide data to bridge the molecular gap between South China and India. Replication studies in other regions of Thailand will definitely be of interest to investigate potential region-specific patterns of variant RHD allele distribution.

It is important to pay attention to the last six genotypes reported in Table 3, including a total of 28 samples. Indeed, an apparent wild-type $R H D$ allele was found in
23/129 samples (17.8\%), while they were found to be weak D serologically. It is interesting to compare these data with those reported recently in the Indian population, where $37 / 223$ serologically weak D samples (16.6\%) were found to carry at least one apparent wild-type $R H D$ gene copy [29]. On the basis of these observations and by considering that replication studies in both populations yield comparable results, we suggest that more complete RHD gene investigation, including yet unexplored regions of regulation and introns, should be carried out to further identify potential functional variations altering $\mathrm{D}$ antigen expression. Discrepancy in the genotype-phenotype correlation in the remaining five samples (i.e., RHD*01EL.01/RHD*01N.01 [ $n=1]$; RHD*01EL.01/ $R H D^{*} 01 N .03[n=1]$, and $R H D^{*} 01 N .01 / R H D^{*} 01 N .01$ $[n=3])$ remains to be elucidated by additional serological testing in the same samples first.

Overall, we report the molecular basis of variant $\mathrm{D}$ expression in Thai blood donors for the first time. Beyond the fundamental interest of this study in terms of population genetics and molecular epidemiology of the Rh blood group system, the next step will be the design and implementation of a population-specific screening test based on our data to help clinicians in their current practice to further improve blood transfusion safety.

\section{Acknowledgement}

The authors are grateful to the Thai blood donors who contributed their blood samples for this study.

\section{Statement of Ethics}

The project was approved by Mahidol University Central Institutional Review Board (MU-CIRB 2018/104.1805) and Research Ethics Committee, NBC, Thai Red Cross Society (COA No. NBC 13/2018). All participants provided their informed consent.

\section{Disclosure Statement}

The authors declare no conflicts of interest.

\section{Funding Sources}

This study was supported by the Faculty of Medical Technology, Mahidol University, Bangkok, Thailand; the Etablissement Français du Sang (EFS) - Bretagne, and the Institut National de la Santé et de la Recherche Médicale (INSERM), France. 


\section{References}

1 Colin Y, Chérif-Zahar B, Le Van Kim C, Raynal V, Van Huffel V, Cartron JP. Genetic basis of the RhD-positive and $\mathrm{RhD}$-negative blood group polymorphism as determined by Southern analysis. Blood. 1991 Nov;78(10): 2747-52.

2 Klein HG, Anstee DJ. The Rh blood group system (and LW). In: Klein HG, Anstee DJ, editors. Mollison's blood transfusion in clinical medicine. 11th ed. Malden (MA): Oxford, Blackwell Publishing; 2005. pp. 163-208.

3 Wagner FF, Gassner C, Müller TH, Schönitzer D, Schunter F, Flegel WA. Molecular basis of weak D phenotypes. Blood. 1999 Jan;93(1): 385-93.

4 Singleton BK, Green CA, Avent ND, Martin PG, Smart E, Daka A, et al. The presence of an RHD pseudogene containing a 37 base pair duplication and a nonsense mutation in africans with the Rh D-negative blood group phenotype. Blood. 2000 Jan;95(1):12-8.

5 Wagner FF, Frohmajer A, Ladewig B, Eicher NI, Lonicer CB, Müller TH, et al. Weak D alleles express distinct phenotypes. Blood. 2000 Apr;95(8):2699-708.

6 Shao CP, Maas JH, Su YQ, Köhler M, Legler TJ. Molecular background of Rh D-positive, $\mathrm{D}$-negative, $\mathrm{D}(\mathrm{el})$ and weak $\mathrm{D}$ phenotypes in Chinese. Vox Sang. 2002 Aug;83(2):156-61.

7 Peyrard T. Use of genomics for decision-making in transfusion medicine. ISBT Sci Ser. 2013;8(1):11-5.

8 Peyrard T. Molecular tools for investigating immunohaematology problems. ISBT Sci Ser. 2015;10 S1:31-8.

9 Müller TH, Wagner FF, Trockenbacher A Eicher NI, Flegel WA, Schönitzer D, et al. PCR screening for common weak D types shows different distributions in three Central European populations. Transfusion. $2001 \mathrm{Jan}$; 41(1):45-52.

10 Silvy M, Simon S, Gouvitsos J, Di Cristofaro J, Ferrera V, Chiaroni J, et al. Weak D and DEL alleles detected by routine $\mathrm{SNaPshot}$ genotyping: identification of four novel RHD alleles. Transfusion. 2011 Feb;51(2):401-11.

11 Fichou Y, Le Maréchal C, Jamet D, Bryckaert L, Ka C, Audrézet MP, et al. Establishment of a medium-throughput approach for the ge- notyping of RHD variants and report of nine novel rare alleles. Transfusion. 2013 Aug; 53(8):1821-8.

12 Haer-Wigman L, Veldhuisen B, Jonkers R, Lodén M, Madgett TE, Avent ND, et al. RHD and RHCE variant and zygosity genotyping via multiplex ligation-dependent probe amplification. Transfusion. 2013 Jul;53(7):155974.

13 Seo MH, Won EJ, Hong YJ, Chun S, Kwon JR, Choi YS, et al. An effective diagnostic strategy for accurate detection of $\mathrm{RhD}$ variants including Asian DEL type in apparently RhD-negative blood donors in Korea. Vox Sang. 2016 Nov;111(4):425-30

14 Wagner FF, Frohmajer A, Flegel WA. RHD positive haplotypes in D negative Europeans. BMC Genet. 2001;2(1):10.

15 Kim JY, Kim SY, Kim CA, Yon GS, Park SS. Molecular characterization of D- Korean persons: development of a diagnostic strategy. Transfusion. 2005 Mar;45(3):345-52.

$16 \mathrm{Xu}$ Q, Grootkerk-Tax MG, Maaskant-van Wijk PA, van der Schoot CE. Systemic analysis and zygosity determination of the RHD gene in a $\mathrm{D}$-negative Chinese Han population reveals a novel D-negative RHD gene. Vox Sang. 2005 Jan;88(1):35-40.

17 Fongsarun J, Nuchprayoon I, Yod-in S, Kupatawintu P, Kidprasirt C. Blood groups in Thai blood donors. Thai J Hematol Transfus Med. 2002;12:277-86.

18 Makechay S, Siripongsanusit A, Sakuldamrongpanich T. Incidence of Partial D Categories in Thai Blood Donors. J Hematol Transfus Med. 2010;20:105-12.

19 Srijinda S, Suwanasophon C, Visawapoka U, Pongsavee M. RhC Phenotyping, adsorption/ elution test, and SSP-PCR: the combined test for D-elute phenotype screening in Thai RhD-negative blood donors. ISRN Hematol. 2012;2012:358316

20 Jongruamklang P, Gassner C, Meyer S, Kummasook A, Darlison M, Boonlum C, et al. Matrix-assisted laser desorption/ionization time-of-flight mass spectrometry analysis of 36 blood group alleles among 396 Thai samples reveals region-specific variants. Transfusion. 2018 Jul;58(7):1752-62.
21 Burin des Roziers N, Squalli S. Removing IgG antibodies from intact red cells: comparison of acid and EDTA, heat, and chloroquine elution methods. Transfusion. 1997 May;37(5): 497-501.

22 Fichou Y, Le Maréchal C, Bryckaert L, Dupont I, Jamet D, Chen JM, et al. A convenient qualitative and quantitative method to investigate RHD-RHCE hybrid genes. Transfusion. 2013 Nov;53(11 Suppl 2):2974-82.

23 Fichou Y, Gehannin P, Corre M, Le Guern A, Le Maréchal C, Le Gac G, et al. Extensive functional analyses of RHD splice site variants: insights into the potential role of splicing in the physiology of Rh. Transfusion. 2015 Jun;55(6 Pt 2):1432-43.

24 Silvy M, Chapel-Fernandes S, Callebaut I, Beley S, Durousseau C, Simon S, et al. Characterization of novel RHD alleles: relationship between phenotype, genotype, and trimeric architecture. Transfusion. 2012 Sep;52(9): 2020-9.

25 Okuda H, Kawano M, Iwamoto S, Tanaka M, Seno T, Okubo Y, et al. The RHD gene is highly detectable in $\mathrm{RhD}$-negative Japanese donors. J Clin Invest. 1997 Jul;100(2):373-9.

26 Kulkarni SS, Gogri H, Parchure D, Mishra G, Ghosh K, Rajadhyaksha S, et al. RHD-positive alleles among $\mathrm{D}-\mathrm{C} / \mathrm{E}+$ individuals from India. Transfus Med Hemother. 2018 May; 45(3):173-7.

27 Omi T, Takahashi J, Tsudo N, Okuda H, Iwamoto S, Tanaka M, et al. The genomic organization of the partial D category DVa: the presence of a new partial D associated with the DVa phenotype. Biochem Biophys Res Commun. 1999 Jan;254(3):786-94.

28 Ji YL, Luo H, Wen JZ, Haer-Wigman L, Veldhuisen B, Wei L, et al. RHD genotype and zygosity analysis in the Chinese Southern Han $\mathrm{D}+, \mathrm{D}$ - and $\mathrm{D}$ variant donors using the multiplex ligation-dependent probe amplification assay. Vox Sang. 2017 Oct;112(7):660-70

29 Fichou Y, Parchure D, Gogri H, Gopalkrishnan V, Le Maréchal C, Chen JM, et al. Molecular basis of weak D expression in the Indian population and report of a novel, predominant variant RHD allele. Transfusion. 2018 Jun;58(6):1540-9. 